\title{
Organoids as an in vitro model of human development and disease
}

\author{
Aliya Fatehullah ${ }^{1}$, Si Hui Tan ${ }^{1}$ and Nick Barker ${ }^{1,2,3, \star}$
}

\begin{abstract}
The in vitro organoid model is a major technological breakthrough that has already been established as an essential tool in many basic biology and clinical applications. This near-physiological 3D model facilitates an accurate study of a range of in vivo biological processes including tissue renewal, stem cell/niche functions and tissue responses to drugs, mutation or damage. In this Review, we discuss the current achievements, challenges and potential applications of this technique.
\end{abstract}

The successful exploitation of human stem cells for clinical use has long been hampered by our inability to maintain and expand adult stem cells while retaining their multi-lineage potential in vitro. However, advances in our understanding of stem cell niches and the role of key signalling modulators in controlling stem cell maintenance and differentiation have fuelled the development of new 3D in vitro culture technologies that sustain stem-cell-driven formation of near-physiological, selfrenewing tissues called organoids. The term 'organoid' has historically been used loosely to encompass all 3D organotypic cultures derived from primary tissues (either tissue subunits or single cells), embryonic stem cells (ESCs) and induced pluripotent stem cells (iPSCs), established cell lines, as well as whole or segmented organs such as organ explants consisting of multiple tissue types ${ }^{1}$. Here we define an organoid as an in vitro 3D cellular cluster derived exclusively from primary tissue, ESCs or iPSCs, capable of self-renewal and self-organization, and exhibiting similar organ functionality as the tissue of origin. Most of the documented organoid cultures contain functional tissue units that lack the mesenchymal, stromal, immune and neural cells that intersperse the tissue in vivo. These organoids rely on artificial extracellular matrices (ECM) to facilitate their self-organization into structures that resemble native tissue architecture.

The widespread implementation of organoid-based technologies across academia and industry is testament to their importance as near-physiological models for use in both basic and translational research. Unlike more traditional in vitro cultures, organoids are similar to primary tissue in both their composition and architecture, harbouring small populations of genomically stable, self-renewing stem cells that give rise to fully differentiated progeny comprising all major cell lineages at frequencies similar to those in living tissue. Another advantage is that organoids can be expanded indefinitely, cryopreserved as biobanks and easily manipulated using techniques similar to those established for traditional 2D monolayer culture. Finally, the fact that primary-tissue-derived organoids lack mesenchyme/stroma provides a reductionist approach for studying the tissue type of interest without confounding influences from the local microenvironment. Organoids represent an important bridge between traditional 2D cultures and in vivo mouse/human models, as they are more physiologically relevant than monolayer culture models and are far more amenable to manipulation of niche components, signalling pathways and genome editing than in vivo models (Table 1).

In this Review, we discuss recent developments in murine and human organoid technologies from primary tissues as well as ESCs and iPSCs. We critically appraise the value of organoids as model systems for understanding human development and disease, as well as for clinical applications. We also highlight key challenges that remain to be addressed.

\section{A brief history of organoids}

Researchers have long known of the self-organizing capacity of mammalian cells and have harnessed this ability to generate 3D cultures from primary tissues, but the development of the intestinal organoid culture system in 2009 was a major technological advance for the stem cell field. Unlike previous systems, this new method made use of our knowledge of endogenous intestinal stem cell niche components to deliver a welldefined, stable culture system capable of sustaining the long-term growth of near-physiological epithelia from purified $\mathrm{Lgr}^{+}$stem cells or isolated crypts. The culture system was surprisingly simple, using Matrigel as an ECM substitute, supplemented with growth factors constituting key endogenous niche signals: WNT, a Frizzled/LRP (lipoprotein receptorrelated protein) ligand; Noggin, a BMP (bone morphogenetic protein) inhibitor, to allow for stem cell expansion; R-spondin, an LGR4/5 (leucine-rich repeat-containing G-protein-coupled receptor 4/5) ligand, a WNT agonist to maintain stem cell populations; and EGF (epithelial growth factor), an EGFR ligand, to promote cell proliferation. As such, this culture system is commonly referred to as the R-spondin method. It was used to create 3D structures with distinct crypt-like and villus-like 
Near-physiological model system for studying adult stem cells and tissues in a variety of contexts

Adult stem cells can be propagated in organoids, and specific tissue lineages can be cultured in high purity with minimal contributions from other cell types (for example, fibroblasts and endothelial cells)

Can be propagated for a long time (years) without genomic alterations

Amenable to a wide variety of established experimental techniques

Can be derived from multiple sources: adult and foetal tissues, ESCs and iPSCs

Can generate organoids encompassing a broad range of tissues

Limited amounts of starting material can be expanded for numerous applications

Human diseases that are difficult to model in animals can be studied with patient-derived organoids

Possibility of generating isogenic adult tissue for transplantation in regenerative procedures

Limitations
$\begin{gathered}\text { Lack of native microenvironment precludes studies about interaction of stem } \\ \text { cells with their niches, immune cells, etc. }\end{gathered}$
Limited use in modelling inflammatory responses to infection or drugs due to
absence of immune cells in culture system
Unable to mimic in vivo growth factor/signalling gradients in Matrigel matrix
Unable to mimic biomechanical forces that stem cells encounter in vivo
Relatively rigid ECM could limit drug penetration, hence
hampering the use of organoids in drug screens
understood (for example, the ovary)
Organoids in the same culture are heterogeneous in terms of viability, size and
shape, impeding phenotype screens
Organoid cultures depend on mouse-sarcoma-derived Matrigel, which
precludes transplantation of organoids into humans

domains bordering a central lumen containing dead cells extruded from the constantly renewing epithelial layer ${ }^{2}$ (Table 2 and Fig. 1). Remarkably, these organoids faithfully recapitulated the in vivo tissue architecture and contained the full complement of stem, progenitor and differentiated cell types. The system was subsequently adapted for generating human intestinal organoids, as well as organoids from other organs harbouring $\mathrm{Lgr5}^{+}$stem cells, including the colon, stomach and liver ${ }^{3-7}$.

\section{Organoids from primary tissue}

Organoids have been successfully generated from many regions of the mouse gastrointestinal tract, ranging from the tongue through to the colon (Table 2). Spherical lingual organoids comprising a multilayer keratinized epithelium and stratum corneum were generated from isolated cells expressing Bmil, an established marker of adult stem cells ${ }^{8}$. More recently, single cells expressing Lgr5 or Lgr6, two other well-known adult stem cell markers, successfully gave rise to functional taste bud organoids ${ }^{9}, 10$. Other studies have described the generation of ductal and lobular-type salivary gland organoids from salivary gland primary sphere cells plated in a mixture of Matrigel and collagen ${ }^{11}$. In the oesophageal organoid cultures, the addition of Gastrin to the R-spondin-based culture media helped generate organoids that allowed researchers to identify cells involved in the self-renewal of oesophageal epithelium ${ }^{12}$. Gastric organoids from the pylorus and corpus regions can also be grown using a variation of this R-spondin-based culture method. However, differentiation of the proliferative organoids requires withdrawal of WNT3A and FGF10 (fibroblast growth factor 10) from pyloric cultures, or WNT3A, FGF10 and Noggin from corpus cultures ${ }^{5,6}$.
Complement with organotypic culture system, or co-culture with other cell types such as stromal cells ${ }^{15}$ or immune cells

Apply microfluidic technologies to generate concentration gradients

Novel substrates and ECM factors are being identified to model such interactions in vitro ${ }^{50-52}$

Devise ways to vary physical attributes of ECM such as composition, porosity and stiffness

Screen for small-molecule modulators of key signalling pathways and specific hormones as potential culture components

Organoids can be tracked individually by live or time-lapse imaging

More defined ECMs that support organoid growth are being developed to comply with regulations for transplantation into humans

Establishing 3D organoids from other gastrointestinal epithelial organs has proven more challenging, requiring tissue-specific modifications that reflect the individual niche requirements and lineage commitment factors for the resident stem cell populations and their progeny (Fig. 1). The addition of various modulators of WNT (for example, CHIR99021, which inhibits GSK3 $\beta$ (glycogen synthase kinase $3 \beta$ )) and Notch signalling pathways (for example, valproic acid, which inhibits histone deacetylase) has helped enrich and maintain the stem cell population, resulting in a more successful organoid culture ${ }^{13}$. A particularly exciting recent development has been the long-term growth of organoids from the adult liver and pancreas. Following acute injury, the pathways involved in bile duct and islet formation/regeneration were reactivated, facilitating the identification of the adult stem cell pool ${ }^{714}$. Liver bile duct fragments plated into Matrigel and supplemented with EGF, R-spondin, HGF (hepatocyte growth factor), FGF10 and nicotinamide generated cystic organoids. These organoids contained cells expressing biliary ductal markers, which could be differentiated to a functional hepatocyte lineage by the inhibition of the Notch and TGF- $\beta$ signalling pathways? Adult tissue from pancreatic ducts was observed to generate budding cyst-like organoids when supplemented with EGF, R-spondin, FGF10 and nicotinamide. Isolated acinar cells could generate smaller, shortlived duct-like organoids, whereas cultures derived from endocrine cells were viable for 30 days but were non-proliferative. Importantly, engraftment of these pancreatic organoids under the kidney capsule resulted in the formation of functional pancreatic tissue containing ductal, endocrine and acinar cells, providing strong evidence that pancreatic stem cell potential resides in the adult ductal compartment ${ }^{14}$. In addition to adult 
Table 2 Organoid models from various organs and ESCs/iPSCs

\begin{tabular}{|c|c|c|c|c|c|c|}
\hline Tissue & Source & Organoid morphology & Cell types in organoid & $\begin{array}{c}\text { Downstream applica- } \\
\text { tions tested }\end{array}$ & $\begin{array}{l}\text { Types of human } \\
\text { diseases modelled }\end{array}$ & Refs \\
\hline Lingual & $\begin{array}{l}\text { Mouse lingual } \\
\text { stem cells }\end{array}$ & $\begin{array}{l}\text { Spherical and budding } \\
\text { organoids }\end{array}$ & $\begin{array}{c}\text {-Stratum corneum } \\
\text {-Keratin } 5 / 14^{+} \text {stem/progenitor cells } \\
\text {-Granular cells of intermediate epithelial origin }\end{array}$ & $\begin{array}{l}\text {-Tamoxifen inductions } \\
\text {-Lineage tracing } \\
\text {-Engraftment studies }\end{array}$ & Lingual carcinoma & 8 \\
\hline Taste bud & $\begin{array}{l}\text { Murine circum- } \\
\text { vallate tissue }\end{array}$ & $\begin{array}{l}\text { Spherical and budding } \\
\text { organoids }\end{array}$ & $\begin{array}{c}\text {-Lgr } 5^{+} \text {stem cells } \\
\text {-Lgr6 } 6^{+} \text {stem/progenitor cells } \\
\text {-UEA/K8+ intragemmal taste bud cells } \\
\text {-Gustducin-expressing, T1R3-expressing } \\
\text { mature taste receptor cells } \\
\text {-Sox9+ proliferative cells in budding regions }\end{array}$ & $\begin{array}{l}\text {-Functional assays } \\
\text {-Gene expression analysis } \\
\text {-Cell cycle analysis }\end{array}$ & - & 9,10 \\
\hline $\begin{array}{l}\text { Salivary } \\
\text { gland }\end{array}$ & $\begin{array}{l}\text { Primary mouse } \\
\text { cells }\end{array}$ & $\begin{array}{l}\text {-Ductal branching organoids } \\
\text {-Lobular spherical organoids }\end{array}$ & $\begin{array}{l}\text {-CD24 }{ }^{\text {hi }} / C D 29^{\text {hi }} \text { stem cells } \\
\text {-Cytokeratin } 7 / 18^{+} \text {duct cells. } \\
\text {-Aquaporin5-expressing acinar cells }\end{array}$ & $\begin{array}{l}\text {-Gene expression analysis } \\
\text {-Transplantation studies }\end{array}$ & Hyposalivation & 11 \\
\hline Oesophagus & $\begin{array}{l}\text { Mouse/human } \\
\text { adult oesopha- } \\
\text { geal tissue }\end{array}$ & $\begin{array}{c}\text {-Spherical multilayered } \\
\text { organoids } \\
\text {-Human Barrett's oesophagus } \\
\text { organoids: budding mor- } \\
\text { phology }\end{array}$ & $\begin{array}{l}\text {-CK } 14^{+}, \mathrm{p} 63^{+} \text {small basal cells in the outer } \\
\text { layer, large basal cells in the middle layer, and } \\
\text { CK13 } 13^{+} \text {cells in the central keratinized layer } \\
\text {-Integrin } \alpha_{6} / \beta_{4}^{\text {low }} \text { differentiated and } \\
\text { integrin } \alpha_{6} / \beta_{4}^{\text {hi }} \text { stem cells } \\
\text {-Barrett's oesophageal organoids express goblet } \\
\text { cells following differentiation. }\end{array}$ & $\begin{array}{l}\text {-Tamoxifen inductions } \\
\text {-Gene expression analysis }\end{array}$ & Barrett's oesophagus & 3,12 \\
\hline Stomach & $\begin{array}{l}\text {-Mouse/human } \\
\text { adult tissue } \\
\text {-Mouse/human } \\
\text { ESCs } \\
\text {-Human iPSCs }\end{array}$ & $\begin{array}{l}\text {-Pylorus and corpus: spheri- } \\
\text { cal organoids } \\
\text {-FGF10-driven budding } \\
\text { events in pyloric organoids }\end{array}$ & $\begin{array}{l}\text {-Pylorus: Lgr5+ stem cells, mucous neck cells, } \\
\text { pit cells, enteroendocrine cells } \\
\text {-Corpus: chief cells, mucous neck cells, pit } \\
\text { cells }\end{array}$ & $\begin{array}{l}\text {-Microinjection and infection } \\
\text { model for H. pylori } \\
\text {-Transcriptome profiling } \\
\text {-Co-culture with mesenchy- } \\
\text { mal cells } \\
\text {-Adeno/retroviral } \\
\text { transfections }\end{array}$ & $\begin{array}{l}\text {-H. pylori infection } \\
\text {-Cancer }\end{array}$ & $\begin{array}{l}5,6,23 \\
24,32 \\
33,70 \\
80-82\end{array}$ \\
\hline Intestine & $\begin{array}{l}\text {-Adult tissue } \\
\text {-Mouse/human } \\
\text { ESCs } \\
\text {-Human iPSCs }\end{array}$ & $\begin{array}{l}\text {-Normal tissue: branching } \\
\quad \text { organoids } \\
\text {-Diseased tissue: cystic and } \\
\text { other morphologies }\end{array}$ & $\begin{array}{l}\text {-Crypt-like domain contains Lgr5+ stem cells } \\
\text { and paneth cells } \\
\text {-Villus-like domain harbours villin+ cells } \\
\text {-Enteroendocrine and goblet cells scattered } \\
\text { throughout organoid }\end{array}$ & $\begin{array}{l}\text {-Transplantation studies } \\
\text {-CRISPR/Cas9 gene editing } \\
\text {-Cancer mutations } \\
\text {-Transcriptome profiling }\end{array}$ & $\begin{array}{l}\text {-Cancer } \\
\text {-Cystic fibrosis } \\
\text {-Infection model for } \\
\text { viral and bacterial } \\
\text { infection }\end{array}$ & $\begin{array}{c}2-4,13, \\
17-21,30 \\
31,53-55 \\
57-59 \\
61-64,72 \\
76,77,79 \\
83,84\end{array}$ \\
\hline Colon & $\begin{array}{l}\text {-Adult tissue } \\
\text {-Mouse/human } \\
\text { iPSCs }\end{array}$ & $\begin{array}{l}\text {-Normal tissue: budding } \\
\quad \text { organoids } \\
\text {-Diseased tissue: cystic } \\
\text { morphologies }\end{array}$ & $\begin{array}{l}\text {-Lgr } 5^{+} \text {stem cells } \\
\text {-Enteroendocrine cells } \\
\text {-Goblet cells } \\
\text {-Enterocytes }\end{array}$ & $\begin{array}{l}\text {-Transplantation studies } \\
\text {-Transcriptome profiling } \\
\text {-Biobank }\end{array}$ & $\begin{array}{l}\text {-IBD } \\
\text {-Cancer }\end{array}$ & $\begin{array}{l}3,21,22 \\
56,60,65\end{array}$ \\
\hline Liver & $\begin{array}{l}\text {-Mouse adult } \\
\text { tissue } \\
\text {-Human iPSCs }\end{array}$ & $\begin{array}{l}\text {-Mouse organoids: spherical } \\
\text {-Human organoids: cystic }\end{array}$ & $\begin{array}{c}- \text { Lgr } 5^{+} \text {stem cells and bile duct cells } \\
\text {-Hepatocytes observed after inhibition of Notch } \\
\text { \& TGF- } \beta \text { pathways } \\
\text {-Human organoids contain Lgr5+ stem cells, } \\
\text { ductal cells and hepatocytes } \\
\text {-Cholangiocyte organoids express differentiation } \\
\text { markers such as apical sodium-dependent bile } \\
\text { acid transporter, secretin receptor, cilia and } \\
\text { CFTR. }\end{array}$ & $\begin{array}{l}\text {-Transplantation studies } \\
\text {-Transcriptome profiling } \\
\text {-Adenoviral transduction } \\
\text {-Whole-genome sequencing } \\
\text {-CFTR functional assays }\end{array}$ & $\begin{array}{l}\text {-Alagille syndrome } \\
\text {-Cystic fibrosis }\end{array}$ & $\begin{array}{c}7,25 \\
34-36,85\end{array}$ \\
\hline Pancreas & $\begin{array}{l}\text { Mouse/human } \\
\text { adult tissue }\end{array}$ & $\begin{array}{l}\text {-E10.5 tissue: branching } \\
\text { organoids } \\
\text {-Adult tissue: budding } \\
\text { organoids }\end{array}$ & $\begin{array}{l}\text {-Ductal organoids contain Lgr } 5^{+} \text {stem cells } \\
\text {-Organoids from E10.5 murine pancreas can be } \\
\text { differentiated into exocrine and endocrine cells }\end{array}$ & $\begin{array}{l}\text {-Engraftment studies } \\
\text {-Lineage tracing } \\
\text {-Adenoviral transduction } \\
\text {-Transcriptome profiling }\end{array}$ & Cancer & $14,26,66$ \\
\hline Prostate & $\begin{array}{l}\text { Mouse/human } \\
\text { adult tissue }\end{array}$ & $\begin{array}{l}\text {-Normal tissue: spherical } \\
\text {-Diseased tissue: branching } \\
\text { similar to morphology of can- } \\
\text { cerous organoids }\end{array}$ & $\begin{array}{l}\text {-Cytokeratin } 5 / \mathrm{p} 63^{+} \text {basal cells } \\
\text {-Cytokeratin } 8^{+} \text {luminal cells. } \\
\text { Lgr } 4 / \text { Lgr } 5^{+} \text {cells }\end{array}$ & $\begin{array}{l}\text {-Tamoxifen inductions } \\
\text {-Lentiviral infections }\end{array}$ & Cancer & $27,28,86$ \\
\hline Lung & $\begin{array}{l}\text {-Mouse foetal } \\
\text { pulmonary } \\
\text { cells } \\
\text {-Human ESCs/ } \\
\text { iPSCs }\end{array}$ & Spherical organoids & $\begin{array}{l}\text {-Uniform proximal Sox2 and distal Sox9 } \\
\text { expression } \\
\text {-Proximal-like domains included basal, cili- } \\
\text { ated and club cells that were surrounded by } \\
\text { smooth-muscle actin }\end{array}$ & $\begin{array}{l}\text {-Transplantation studies } \\
\text {-Cancer mutations } \\
\text {-RNA sequencing }\end{array}$ & Cystic fibrosis & $15,16,37$ \\
\hline Retina & Mouse ESCs & Spherical organoids & $\begin{array}{c}\text {-Outer layer recapitulates retinal pigment } \\
\text {-Invaginated layer resembles retinal optic cup } \\
\text { containing photoreceptors, ganglion cells, bipo- } \\
\text { lar cells and Muller glia }\end{array}$ & - & - & 38 \\
\hline Inner ear & Mouse ESCs & Budding organoids & $\begin{array}{c}\text {-Functional prosensory vesicles } \\
\text {-Otic vesicles also generate functional inner-ear } \\
\text { hair cells }\end{array}$ & - & - & 41,87 \\
\hline Brain & $\begin{array}{l}\text {-Mouse/human } \\
\text { ESCs } \\
\text {-Human iPSCs } \\
\text {-Patient skin } \\
\text { fibroblasts }\end{array}$ & $\begin{array}{l}\text {-Early-stage spherical } \\
\text { organoids } \\
\text {-Later-stage budding } \\
\text { organoids }\end{array}$ & $\begin{array}{l}\text {-Early-stage organoids contain continuous } \\
\text { neuroepithelia } \\
\text {-Cerebral cortical regions contain outer radial } \\
\text { glia and cerebral cortical neurons }\end{array}$ & $\begin{array}{l}\text {-Transfection } \\
\text {-Transcriptome profiling } \\
\text {-Electrical excitation }\end{array}$ & $\begin{array}{l}\text {-Autism } \\
\text {-Microcephaly }\end{array}$ & $\begin{array}{l}39,40 \\
73,88\end{array}$ \\
\hline Kidney & Human iPSCs & $\begin{array}{l}\text {-Early-stage spherical } \\
\text { organoids } \\
\text {-Differentiated stages have } \\
\text { budding morphology }\end{array}$ & $\begin{array}{l}\text { Differentiated organoids exhibit segmentation } \\
\text { as ducts, tubules and glomeruli }\end{array}$ & $\begin{array}{l}\text { Toxicity screening in } \\
\text { response to cisplatin }\end{array}$ & - & 42 \\
\hline
\end{tabular}

A summary of the organs that have seen success using the organoid model, including descriptions of the typical morphologies and cell types that have been observed, and the various diseases along with the associated techniques that have been modelled in the organoids. ESCs, embryonic stem cells; iPSCs, induced pluripotent stem cells. 
a Harvest tissue/obtain tissue biopsy

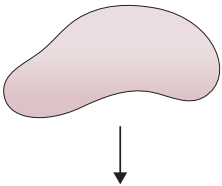

b Dissociate tissue into functional units

Enrich for stem cells

Spherical/cyst-like
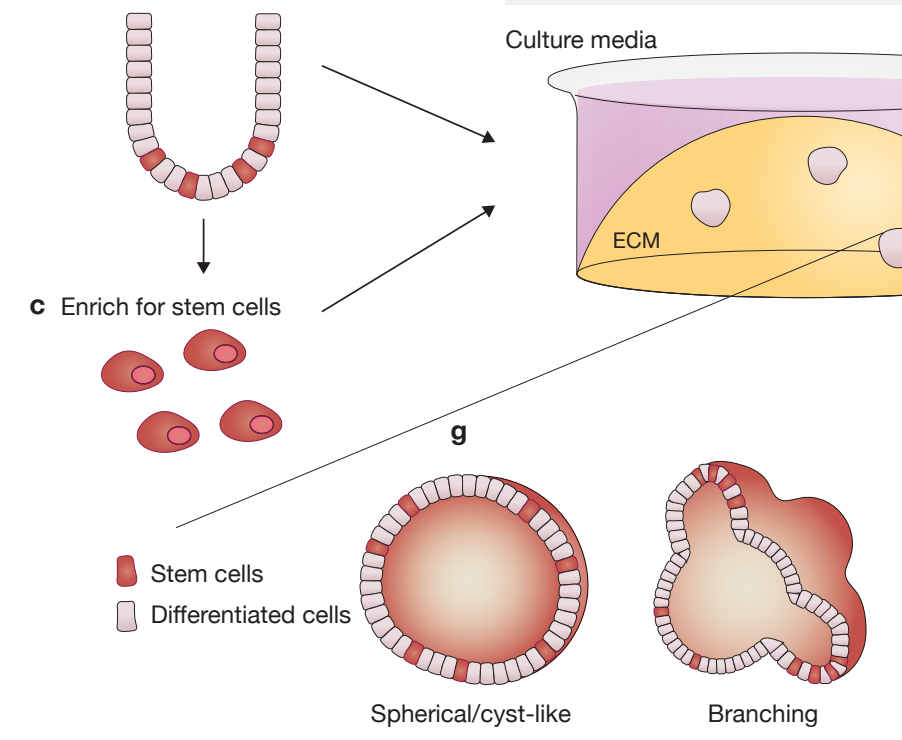

\section{f}

$\begin{array}{ccc} & \text { Niche factors } \\ \text { R-spondin } & \text { EGF } & \text { Noggin } \\ \text { WNT3A } & \text { FGF10 } & \text { Activin A } \\ \text { Retinoic acid } & \text { HGF } & \text { Gastrin } \\ \text { GSK3 } \beta \text { inhibitors } & \text { HDAC inhibitors } & \text { p38 inhibitors } \\ \text { TGF- } \beta \text { inhibitors } & \text { ROCK inhibitors } & \end{array}$

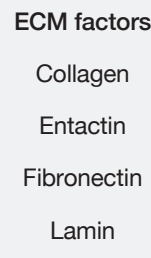

ECM factors

d ESCs/iPSCs

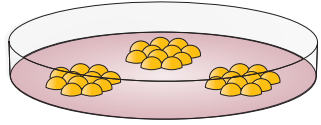

Lamin
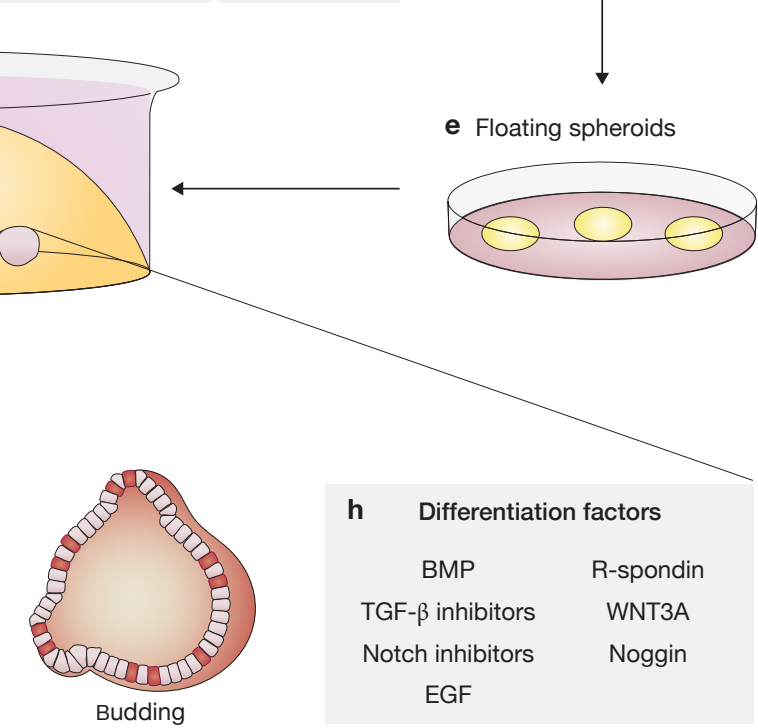

Figure 1 Organoid generation and culture from primary tissue and ESCs/iPSCs. (a-c) Organoids can be generated from primary tissue (a) that is dissociated into functional sub-tissue units containing stem cells (b). These are further digested into single cells and FACSsorted to enrich for stem cells (c). Both the functional units and single stem cells can give rise to organoids under the appropriate culture conditions. (d,e) Organoids derived from ESCs and iPSCs (d) undergo directed differentiation towards the desired germ lineage, eventually generating floating spheroids (e) that are subsequently embedded in ECM to initiate organoid culture. Organoids are typically cultured in an

tissues, primary fetal tissue can also be used to generate organoids. Lung organoids were generated by manipulating VEGF (vascular endothelial growth factor), $\mathrm{SHH}$ (sonic hedgehog) and FGF signalling pathways in murine embryonic pulmonary cells that exhibited branching morphogenesis and sacculation-like structures. As the process is remarkably similar to lung development in vivo, the pulmonary organoid culture provides a valuable in vitro tool for studying lung development ${ }^{15,16}$.

Perhaps the most clinically relevant breakthrough has been the development of culture systems sustaining long-term growth and expansion of human organoids in vitro. In 2011, the first human organoids derived from both healthy and diseased oesophageal, intestinal and colonic epithelia were grown using the mouse R-spondin culture system supplemented with Gastrin, FGF10, TGF- $\beta$, MAP kinase inhibitors and nicotinamide, with other groups reporting similar success $^{3,4,17-22}$. It is now possible to culture organoids from human gastric tissue by varying concentrations of Gastrin and adding inhibitors of the TGF- $\beta$ pathway to promote stem/progenitor cell maintenance ${ }^{23,24}$. Human liver organoids have also been successfully derived from healthy biopsy samples. These cultures contained bi-potent stem cells that generated exclusively ductal epithelia under standard culture conditions, but retained the potential to produce mature hepatocytes when extracellular matrix (ECM) surrounded by culture media supplemented with niche factors specific to the organoid type. (f) Common niche and ECM factors are listed, including factors constituting the ' $R$-spondin method' (see main text for further details). (g) Stem cells are maintained and perpetuated in organoids, continually giving rise to differentiated progeny. Typical morphologies are classified as spherical, branching or budding. (h) Organoids can either differentiate spontaneously or be induced to differentiate towards desired lineages or cell types by adding suitable differentiation factors (left column) and/or withdrawing factors that promote stemness (right column).

differentiated in the presence of Notch inhibitors, FGF19, BMP7 and dexamethasone. Significantly, these organoids were capable of functional engraftment following transplantation into mice with acute liver damage ${ }^{25}$. Employing a specific cocktail of WNT pathway activators and TGF- $\beta$ pathway inhibitors, Boj et al. reported limited success in culturing human pancreatic organoids that could be maintained for up to six months and successfully cryopreserved ${ }^{26}$. Propagation of mouse and human prostate epithelium as multi-layered spherical organoids harbouring bi-potent progenitor cells capable of differentiation towards both basal (outer layer) and luminal (inner layer) lineages has also been achieved using media supplemented with BMP receptor inhibitors ${ }^{27}$. Such human prostate organoids comprising basal and luminal cell lineages can be generated from single normal human luminal and basal cells. Furthermore, tumour biopsies and circulating tumour cells from patients can generate organoids whose histologies mirror those of the primary tumours ${ }^{27,28}$.

\section{Organoids from ESCs and iPSCs}

The use of murine and human ESC and iPSC lines to generate organoids circumvents the limited availability of high-quality human primary material, but requires detailed knowledge of the factors involved in 
germ layer and subsequent lineage specification to perform directed differentiation. The use of iPSC lines requires an additional step compared to ESCs, as somatic cells first have to be converted into iPSCs through the expression of OCT4, KLF4, SOX2 and MYC ${ }^{29}$. Thereafter, ESCs and iPSCs are exposed to germ layer and tissue-specific patterning factors, followed by embedding in Matrigel to facilitate the development of 3D architecture, and treatment with differentiation factors to produce the intended organoids.

For endodermal tissues, TGF- $\beta$ signalling is stimulated in ESCs and iPSCs to form definitive endoderm, which then differentiates into the relevant segment of the embryonic gut based on culture conditions. To generate intestinal organoids, FGF4 and WNT3A were applied to definitive endoderm cells from human ESCs and iPSCs to promote hindgut and intestinal fate, whereas another study cultured definitive endoderm from murine and human ESCs in fibroblast-conditioned

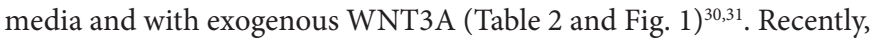
Noguchi et al. ${ }^{32}$ used murine ESCs to generate stomach organoid cultures that recapitulate the features of mature gastric cells found both in the corpus and antrum. By inducing Barx1 expression and concomitantly manipulating the sonic hedgehog $(\mathrm{SHH})$ and WNT signalling pathways, the authors were able to differentiate the definitive endoderm into foregut, and eventually establish near-physiological gastric organoids capable of secreting pepsinogen $\mathrm{c}$ and gastric acid, and displaying rudimentary peristaltic contractions. In contrast, a similar endeavour to generate gastric organoids from human ESCs and iPSCs by manipulating FGF, WNT, BMP, retinoic acid and EGF signalling pathways only reproduced cell types from the gland, pit and neck regions of the antral stomach, but lacked corpus cell lineages ${ }^{33}$. Limited success was also reported for the liver, when iPSC-derived liver buds that also contained human endothelial and mesenchymal cells were cultured short-term before transplantation to generate vascularized, functional liver tissue $\mathrm{e}^{34}$. Recently, human iPSC-derived functional cholangiocyte organoids have also been generated by the modulation of the Activin A and Notch signalling pathways ${ }^{35,36}$. For the lung, human ESC-derived endoderm was supplemented with Hedgehog pathway agonist to drive the sequential commitment towards foregut endoderm and finally spherical epithelial organoids expressing both proximal and distal lung markers observed during branching morphogenesis in vivo. However, no branching was observed in the organoid cultures, unlike those derived from primary fetal tissue ${ }^{15,16,37}$

In ectoderm-derived organoids, ESCs and iPSCs are induced to form embryoid body (EB)-like aggregates, which are then guided towards neural or non-neural fate after ectodermal specification. In the former, mouse ESCs have been used in combination with defined ECM components to generate region-specific retinal organoid cultures resembling the retinal pigment (outer shell) and embryonic optic cup (invaginated) structures $^{38}$. Additionally, two distinct but related methods have been developed to culture brain organoids from murine ESCs, human ESCs and iPSCs ${ }^{38-40}$. The SFEBq (serum-free culture of embryoid body-like aggregates, quick) method pioneered by Eiraku et al..$^{38}$ generates organoids reminiscent of the telencephalon, whereas the cerebral organoid system of Lancaster et al. ${ }^{40}$ employs a spinning bioreactor and produces organoids that recapitulate multiple regions of the brain. Nonetheless, brain organoids derived from both methods exhibited discrete cortical layers and proliferating progenitor zones that contained outer radial glial cells in patterns reminiscent of the early stages of human brain development, and contained neurons that were fully functional and capable of electrical excitation ${ }^{38-40}$. The brain organoid cultures are expected to be a valuable in vitro model for studying the intricate and complex processes occurring during human brain development. Furthermore, inner ear organoids from non-neural ectoderm harbouring prosensory cells and hair cells with mechanosensitive properties have been derived from murine ESCs by manipulating the BMP, FGF and TGF- $\beta$ signalling pathways ${ }^{41}$.

Recently, the first successful mesoderm-derived organoids were reported: renal organoids were produced by modulating GSK3 $\beta$ and FGF signalling pathways in human iPSCs, through an intermediate mesodermal state. These organoids recapitulate the morphology and segmentation of human fetal nephrons into ducts, tubules and glomer$\mathrm{uli}^{42}$. The human renal organoids provide a 3D model to study human renal development and disease under well-defined conditions, thus overcoming various limitations of previous models such as 2D monolayers, short-term 3D aggregates and co-cultures with mouse fibroblasts ${ }^{43-46}$.

A notable difference between organoids derived from primary tissue and ESCs/iPSCs is the presence of cell types other than the intended lineage in the latter. This is because the factors used for directed differentiation of ESCs/iPSCs are not completely efficient in driving all the cells towards the lineage of choice, thus many ectodermal and endodermal organoids, such as those of the intestine, stomach and kidney, have reported the limited presence of mesenchymal cell types ${ }^{31,33,42}$.

Despite these impressive technological advances, there are still tissues that remain resistant to organoid culture, but have been successfully cultured in $3 \mathrm{D}$ as whole-tissue explants or organotypic/mechanically supported cultures (for example, skin or ovary) ${ }^{1,47,48}$. Central to the successful propagation of organoid cultures is the understanding of the endogenous stem cell niche and signalling pathways controlling lineage specification in these tissues. Thus, our relatively poor knowledge in these aspects for certain tissues precludes our ability to rationally design a complement of niche factors conducive to generating organoids. Although it can be argued that identifying the stem cells is not critical for culturing primary tissue units, the understanding of the stem cell niche will be crucial for the sustenance and indefinite propagation of cultures. A potential solution to this would be to screen for small-molecule modulators of key signalling pathways and organ-specific hormones as potential culture components supporting organoid growth from organs such as the ovary. Another potential complicating factor for organoid growth from certain tissues may be a strict dependence on growth factor/ signalling gradients for maintaining balanced stem cell renewal and lineage specification. To circumvent this problem, microfluidic technologies could be used to create concentration gradients more comparable to the in vivo situation. Finally, it is evident that in vivo stem cell behaviour and cell differentiation are also heavily influenced by local biomechanical forces such as those resulting from interactions with the extracellular matrix, which are difficult to replicate in vitro ${ }^{49}$. Efforts are underway to screen for substrates and ECM factors that regulate cell behaviour in vitro in the hope that this will lead to more robust organoid culture models for a wider range of tissues ${ }^{50-52}$ (Table 1).

\section{Applications of organoid technology}

The capability to grow near-physiological, self-renewing organoids in culture provides us with an excellent model system for a wide range of both basic research and translational applications. A major advantage 
b Patient-derived tissue subunits

c Omics profiling

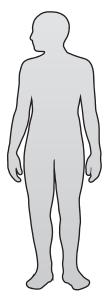

a

g Biobank for academic studies
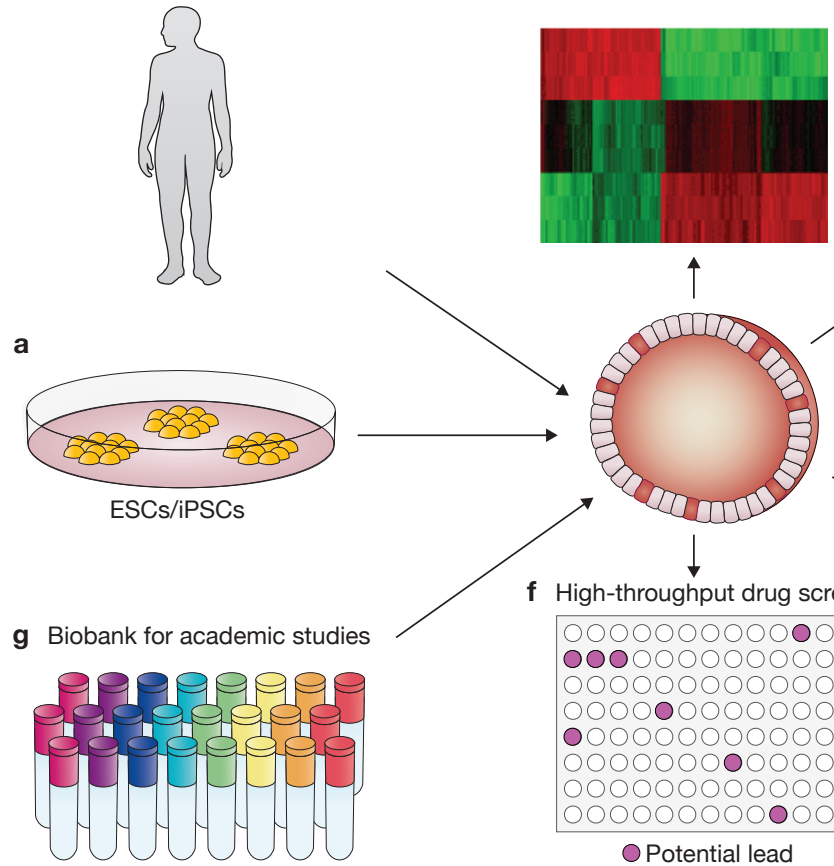

ESCs/iPSCs f $\mathrm{High}$

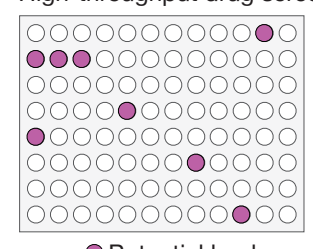

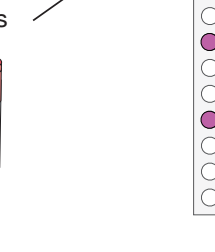

Potential lead d Study host-microbe interaction

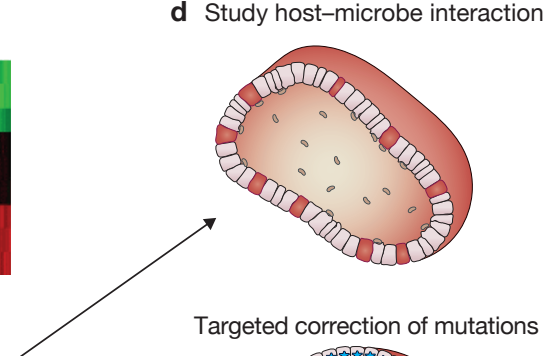

Targeted correction of mutations

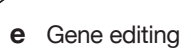
(e.g. CRISPR)
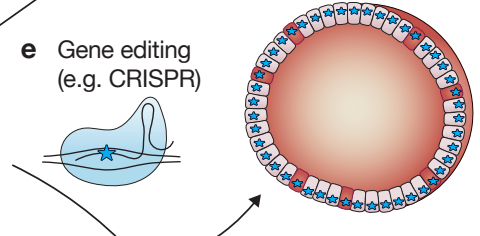

ning

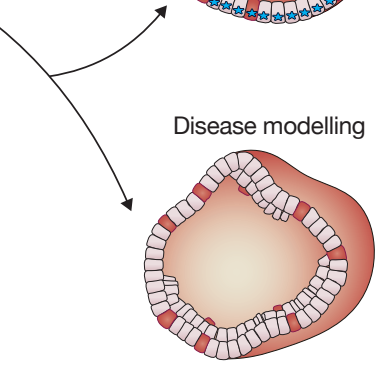

Figure 2 Applications of organoid technology for studying development, homeostasis and diseases. (a,b) Murine- and human-derived organoids from ESCs/iPSCs (a) as well as tissue subunits (b) contribute to the study of development and homeostasis. (c) Genome, transcriptome and proteome profiling of the organoids enables delineation of the contribution of various signalling pathways in development and their dysregulation in disease. (d) Bacterial and viral infections can be studied by injecting

of this system is the ability to greatly expand both tissue-specific stem cells and their differentiated progeny from very limited amounts of starting material such as biopsies, facilitating in-depth analyses of stem cell behaviour, drug screening, disease modelling and genetic screening. Indeed, the intestinal organoids have already been used extensively for analysing stem cell behaviour, identifying niche components, modelling pathogen-epithelia interactions, gene editing, disease modelling and orthotopic transplantation ${ }^{2,4,17-21,53-64}$ (Fig. 2). This success has spurred efforts to create cryopreserved biobanks of healthy and diseased human organoids as a renewable resource that is accessible to researchers worldwide $^{65}$ (Fig. 2g).

Organoids in the study of tissue development and disease. The organoid system allows researchers to intensively study the processes that govern embryonic development, lineage specification and tissue homeostasis, as well as the onset and manifestation of disease. As organoids generated from ESCs, iPSCs and fetal tissues faithfully retain the features of their original developmental stage (Fig. 2a), we can obtain detailed snapshots of embryonic development in a dish as differentiation of the cells is systematically induced. It also delivers invaluable mechanistic insight into the development of stem cells and their niches, while providing an opportunity to monitor their differentiation into mature functional lineages. As an example, fetal pulmonary organoids are being used to determine the signalling interplay between exogenous FGFs essential for endothelial network assembly and the VEGF-A pathway that inhibits the formation of the endothelial network, as well as the crosstalk with the infectious agents into the lumen of organoids. (e) Human disease can be modelled by biochemical and genetic manipulations to identify driver mutations and key signalling pathways. (f) Organoids represent a physiological model that can be used in high-throughput screens for effective drugs and small compounds at the preclinical stage. (g) Importantly, human organoids are being collated and catalogued as openaccess biobanks.

SHH pathway that induces epithelial and endothelial morphogenesis ${ }^{15,16}$. Similarly, the development of tissues such as the stomach, brain and pancreas has been studied through stepwise differentiation of iPSCs and ESCs to organoids. This is achieved by modulating signalling pathways such as WNT, BMP and FGF, thereby elucidating the signalling network that ultimately patterns these tissues ${ }^{33,40,66,67}$. The self-renewing capacity of organoids facilitates the expansion of primary epithelia from very limited starting material for expression profiling studies or for analysing rare cell lineages that are difficult to access in vivo ${ }^{68}$ (Fig. 2c).

Another exciting application of the organoids is modelling hostmicrobe interactions (Fig. 2d). Helicobacter pylori, a causal agent of gastritis and gastric cancer onset in humans, was recently found to efficiently colonize the luminal epithelia of human gastric organoids, resulting in major physiological changes, including an increase in proliferation due to oncogenic CagA and increased $\beta$-catenin signalling ${ }^{23,24,69,70}$. Additionally, some 3D tissue models have been applied to the study of microbial pathogenesis such as haemolytic uremic syndrome caused by Shiga-toxin-producing Escherichia coli ${ }^{71}$. Here, the renal organoids can provide a window to study the cells colonized by the bacterium and the subsequent manifestation in the tissue. As more organ systems become amenable to organoid cultures, the study of bacterial and viral infection and manifestation will allow a greater understanding of the pathogenic mechanisms and subsequently lead to better treatment strategies.

Other studies have employed CRISPR/Cas9-mediated gene editing of healthy organoids to directly evaluate candidate gene function in tissue physiology and carcinogenesis (Fig. 2e). This approach was used to 


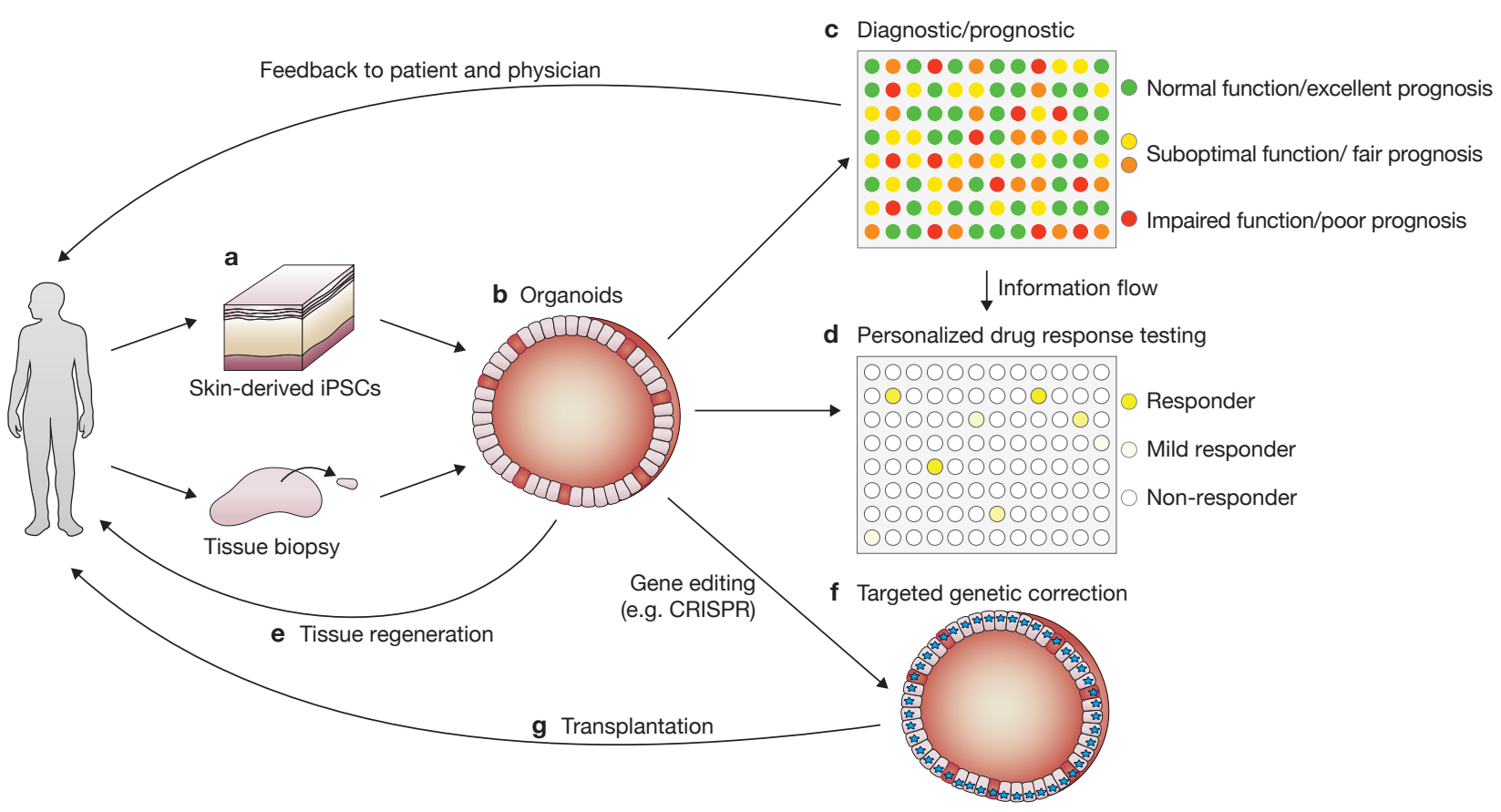

Figure 3 Potential therapeutic and diagnostic uses for organoid technology in personalized medicine. (a) Human iPSC- or tissue-derived organoids can contribute to the design of personalized treatment strategies. (b) The diseased and normal matched organoids can then be used to assess tissue function and prognosis in response to various treatments. (c,d) A wide variety of active drugs and small compounds can be screened for targeting candidate signalling

introduce serial mutations into healthy human colon organoids, converting them into cancer organoids capable of driving in vivo cancer formation following orthotopic or kidney capsule transplantation ${ }^{17,21,72}$. Murine organoids have also been used to model the role of oncogenic Kras in pancreatic neoplasia, accurately recapitulating the human disease. By comparing the gene expression and proteomic profiles of murine organoids with normal or oncogenic Kras, the authors identified signalling pathways and driver genes crucial to the progression of adenocarcinoma ${ }^{26}$.

Certain human diseases have been extremely difficult to model in animals, such as those affecting human brain development. Therefore, patient-derived organoids from adult tissue and iPSCs present an alternative means to dissect the pathologies of these diseases (Fig. 2a,b). For example, studying neural organoids from patients with microcephaly due to loss-of-function mutations in the CDK5RAP2 gene showed that nonfunctional CDK5RAP2 led to premature neural differentiation, resulting in brain hypoplasia ${ }^{40}$. Similarly, transcriptome profiling of organoids derived from iPSCs of autistic patients led to the discovery that these patients exhibit overproduction of GABAergic inhibitory neurons ${ }^{73}$.

Potential of organoids in therapeutics and drug development. Many existing 2D cell lines harbour multiple culture-induced mutations or contamination with other cell lines that limit their value as accurate models for disease modelling or drug screening applications ${ }^{74,75}$. Indeed, over-reliance on such inherently non-physiological models is likely to have contributed to the high failure rate of many drug discovery programs over the past two decades. This has fuelled efforts to develop high-throughput screening methods incorporating the far more stable, pathways (c) to design more effective drug regimens (d) in conjunction with other relevant diagnostic and prognostic factors. (e-g) The organoid cultures can also provide an expansive source of tissue for regeneration (e) and transplantation. Further genetic manipulation of organoids (f) can help restore functionality and normal physiology, and can be transplanted into donors for the re-establishment of physiological function (g).

physiological patient-derived organoids for use in early drug discovery programs and toxicity screens ${ }^{76,77}$. Recently, Ogawa et al. were able to correct CFTR (cystic fibrosis transmembrane conductance regulator) misfolding and translocation to cell membranes in patient-derived cholangiocyte organoids, using inhibitors to reduce misfolding and stabilize the protein ${ }^{36}$. This demonstrates the utility of the organoids for testing and screening novel compounds to treat various conditions (Fig. 2f).

Patient-derived organoids also represent an important resource for developing personalized treatment regimes (Fig. 3a,b). In vitro amplification of patient organoids from disease-site biopsies can deliver sufficient material for deep sequencing to reveal causal mutations, or for in-depth phenotypic profiling to facilitate more tailored treatment regimes (Fig. 3c). The ability to grow matched healthy and diseased organoids from human patients additionally enables clinical screens for drug combinations that selectively target the diseased tissue, helping to identify more effective treatments with minimal side effects (Fig. 3d). Many side effects of anti-cancer drugs can be attributed to acute liver toxicity. One could therefore envisage using hepatic organoids for predicting in vivo liver toxicity of experimental drug combinations before commencing expensive clinical trials ${ }^{78}$. Other clinical applications include the use of disease organoids for predicting acquisition of drug resistance and for developing drugs that effectively target candidate cancer stem cells. Furthermore, in combination with 4D microscopy, organoids can be tracked over time to assess cancer stem cell behaviour and viability in response to active drugs to predict patient outcomes.

Although the development of organoid models represents a major technological breakthrough, the limited presence (if not complete lack) of stromal components, including immune cells, limits their use 
in modelling inflammatory responses to infection or drugs. Another caveat for organoid use in drug-screening programs is potential limitations to drug penetration resulting from the relatively rigid ECM, which could in part be addressed by varying the physical attributes of the ECM (Table 1). Furthermore, variation of ECM physical attributes such as composition, porosity and stiffness should also facilitate modelling of interactions between ECM and the invasive front of tumours, furnishing a means of screening for novel drugs blocking tumourmediated remodelling of ECM during tumour growth and invasion. Unfortunately, organoid cultures are often intrinsically heterogeneous in terms of viability, size and shape, which complicates the analysis of drug toxicity and efficacy. Development of live imaging techniques that facilitate real-time analysis of organoid response and detailed characterization of the heterogeneity within the culture would help to overcome this limitation (Table 1).

Organoids and regenerative medicine. Modern medicine is capable of replacing damaged and/or non-functional tissue with healthy tissue by allogenic transplantation, but a limited supply of healthy donor tissue and the inherent complication of tissue rejection highlight the need for additional tissue sources. Organoid technologies equip researchers with the ability to expand isogenic tissue from miniscule patient biopsies for transplantation use (Fig. 3e). Using iPSC technologies, it is also possible to generate a suite of isogenic or HLA-matched tissue-specific organoids from readily accessible tissue biopsies (for example, skin). Patient organoids harbouring genetic defects can now be repaired using gene-editing technologies to generate healthy isogenic epithelia for use in orthotopic transplantation as an effective treatment regime (Fig. 3f,g). The feasibility of such an approach was demonstrated by Dekkers et al., who employed CRISPR/Cas9 gene editing with patientderived colon organoids to correct germline CFTR mutations, thereby restoring enzymatic function to generate healthy epithelia capable of repopulating diseased tissue following transplantation ${ }^{4}$. Indeed, the capacity of in vitro cultured organoids to repair diseased or damaged tissue in vivo has been demonstrated by studies reporting functional engraftment of orthotopically transplanted organoids in the colon, pancreas and liver ${ }^{4,7,14,25,53,60,79}$. It is expected that patient-derived organoids will be equally proficient in treating a range of human conditions, including ulcerative colitis, Crohn's disease and gastro-oesophageal reflux disease, as well as renal disease and hepatic cirrhosis. Although not currently possible, optimization of pancreatic organoid culture to maintain pancreatic progenitors and functional $\beta$-cells or islets of Langerhans could revolutionize diabetes treatment. Similarly, development of more physiological neural organoids would provide an excellent source of healthy tissue for treating a range of neurodegenerative diseases such as spinal cord injury and Parkinson's disease. Unfortunately, the reliance on mouse sarcoma-derived Matrigel as the artificial 3D matrix in organoid culture precludes the use of human organoids in clinical transplantation due to risks of unforeseen infection and immune/host rejection reactions. Hence, efforts are underway to design more defined ECMs that are compatible with clinical regulations for use in humans (Table 1).

\section{Outlook and future prospects}

Organoids are one of the most accessible and physiologically relevant models to study the dynamics of stem cells in a controlled environment that can be derived from a variety of sources, as exemplified by the epithelial organoids. So far, they have proved to be a robust assay for establishing stem cell identities and niche compositions. In combination with genetic, transcriptome and proteomic profiling, both murine- and human-derived organoids have revealed crucial aspects of development, homeostasis and disease. The progress in generating organoids that faithfully recapitulate the human in vivo tissue composition has extended organoid applications from being just a basic research tool to a translational platform with a wide range of uses. The capacity to indefinitely culture organoids, without introducing genetic variation, makes them a sound model for high-throughput preclinical screenings, designing targeted and personalized therapies, and providing a source of fully functional tissue for regenerative medicine applications.

As interest in organoid technology grows, the commercial development of more standardized, validated organoid culture media will also be valuable in ensuring that the organoid system becomes accessible to a wide range of academic and clinical scientists, thereby helping to maximize its potential. Coupled with a more defined ECM, it is foreseeable that a highly accurate, reproducible culture model could emerge, overcoming current limitations that hinder the technology's transition from bench to bedside.

The organoid technology has synergized well with current methodologies and engendered a wide range of downstream functions and applications, underscoring its broad applicability and potential for manipulation. These features, in conjunction with the physiological relevance of the system, make organoids one of the most exciting and promising technologies that have emerged in recent times for studying human development, disease and therapy.

\section{ACKNOWLEDGEMENTS}

We thank the members of the Barker lab for critical input on the manuscript. A.F., S.H.T. and N.B. are supported by the Agency for Science, Technology and Research $\left(A^{*} \mathrm{STAR}\right)$

\section{ADDITIONAL INFORMATION}

Reprints and permissions information is available online at www.nature.com/ reprints. Correspondence should be addressed to N.B.

\section{COMPETING FINANCIAL INTERESTS}

The authors declare no competing financial interests.

1. Shamir, E. R. \& Ewald, A. J. Three-dimensional organotypic culture: experimental models of mammalian biology and disease. Nat. Rev. Mol. Cell Biol. 15, 647-664 (2014).

2. Sato, T. et al. Single Lgr5 stem cells build crypt-villus structures in vitro without a mesenchymal niche. Nature 459, 262-265 (2009).

3. Sato, T. et al. Long-term expansion of epithelial organoids from human colon, adenoma, adenocarcinoma, and Barrett's epithelium. Gastroenterology 141, 1762-1772 (2011).

4. Dekkers, J. F. et al. A functional CFTR assay using primary cystic fibrosis intestinal organoids. Nat. Med. 19, 939-945 (2013).

5. Barker, N. et al. Lgr5 ${ }^{+v e}$ stem cells drive self-renewal in the stomach and build longlived gastric units in vitro. Cell Stem Cell 6, 25-36 (2010).

6. Stange, D. E. et al. Differentiated Troy ${ }^{+}$chief cells act as reserve stem cells to generate all lineages of the stomach epithelium. Cell 155, 357-368 (2013).

7. Huch, M. et al. In vitro expansion of single Lgr5+ liver stem cells induced by Wnt-driven regeneration. Nature 494, 247-250 (2013).

8. Hisha, H. et al. Establishment of a novel lingual organoid culture system: generation of organoids having mature keratinized epithelium from adult epithelial stem cells. Sci. Rep. 3, 3224 (2013).

9. Aihara, E. et al. Characterization of stem/progenitor cell cycle using murine circumvallate papilla taste bud organoid. Sci. Rep. 5, 17185 (2015).

10. Ren, W. et al. Single Lgr5- or Lgr6-expressing taste stem/progenitor cells generate taste bud cells ex vivo. Proc. Natl Acad. Sci. USA 111, 16401-16406 (2014).

11. Nanduri, L. S. Y. et al. Purification and ex vivo expansion of fully functional salivary gland stem cells. Stem Cell Rep. 3, 957-964 (2014).

12. DeWard, Aaron D., Cramer, J. \& Lagasse, E. Cellular heterogeneity in the mouse esophagus implicates the presence of a nonquiescent epithelial stem cell population. Cell Rep. 9, 701-711 (2014). 
13. Yin, $X$ et al. Niche-independent high-purity cultures of Lgr $5^{+}$intestinal stem cells and their progeny. Nat, Methods 11, 106-112 (2014).

14. Huch, M. et al. Unlimited in vitro expansion of adult bi-potent pancreas progenitors through the Lgr5/R-spondin axis. EMBO J. 32, 2708-2721 (2013).

15. Mondrinos, M. J., Jones, P. L., Finck, C. M. \& Lelkes, P. I. Engineering de novo assembly of fetal pulmonary organoids. Tissue Eng. 20, 2892-2907 (2014).

16. Mondrinos, M.J. et al. Engineering three-dimensional pulmonary tissue constructs. Tissue Eng. 12, 717-728 (2006).

17. Drost, J. et al. Sequential cancer mutations in cultured human intestinal stem cells. Nature 521, 43-47 (2015).

18. Finkbeiner, S. R. et al. Transcriptome-wide analysis reveals hallmarks of human intestine development and maturation in vitro and in vivo. Stem Cell Rep. 4, 1140-1155 (2015).

19. Finkbeiner, S. R. et al. Stem cell-derived human intestinal organoids as an infection model for rotaviruses. MBio 3, e00159-00112 (2012).

20. Forbester, J. L. et al. Interaction of Salmonella enterica serovar Typhimurium with intestinal organoids derived from human induced pluripotent stem cells. Infect. Immun. 83, 2926-2934 (2015).

21. Matano, M. et al. Modeling colorectal cancer using CRISPR-Cas9-mediated engineering of human intestinal organoids. Nat. Med. 21, 256-262 (2015).

22. Jung, P. et al. Isolation and in vitro expansion of human colonic stem cells. Nat. Med. 17, 1225-1227 (2011)

23. Schlaermann, P. et al. A novel human gastric primary cell culture system for modelling Helicobacter pylori infection in vitro. Gut 65, 202-213 (2016).

24. Bartfeld, S. et al. In vitro expansion of human gastric epithelial stem cells and their responses to bacterial infection. Gastroenterology 148, 126-136 (2015).

25. Huch, M. et al. Long-term culture of genome-stable bipotent stem cells from adult human liver. Cell 160, 299-312 (2015).

26. Boj, S. F. et al. Organoid models of human and mouse ductal pancreatic cancer. Cell 160, 324-338 (2015)

27. Karthaus, W. R. et al. Identification of multipotent luminal progenitor cells in human prostate organoid cultures. Cell 159, 163-175 (2014).

28. Gao, D. et al. Organoid cultures derived from patients with advanced prostate cancer. Cell 159, 176-187 (2014).

29. Takahashi, K. \& Yamanaka, S. Induction of pluripotent stem cells from mouse embryonic and adult fibroblast cultures by defined factors. Cell 126, 663-676 (2006).

30. Cao, L. et al. Intestinal lineage commitment of embryonic stem cells. Differentiation 81, 1-10 (2011)

31. Spence, J. R. et al. Directed differentiation of human pluripotent stem cells into intestinal tissue in vitro. Nature 470, 105-109 (2011)

32. Noguchi, T. A. et al. Generation of stomach tissue from mouse embryonic stem cells. Nat. Cell Biol. 17, 984-993 (2015)

33. McCracken, K. W. et al. Modelling human development and disease in pluripotent stem-cell-derived gastric organoids. Nature 516, 400-404 (2014).

34. Takebe, T. et al. Vascularized and functional human liver from an iPSC-derived organ bud transplant. Nature 499, 481-484 (2013).

35. Sampaziotis, F. et al. Cholangiocytes derived from human induced pluripotent stem cells for disease modeling and drug validation. Nat. Biotech. 33, 845-852 (2015).

36. Ogawa, M. et al. Directed differentiation of cholangiocytes from human pluripotent stem cells. Nat. Biotechnol. 33, 853-861 (2015).

37. Dye, B. R. et al. In vitro generation of human pluripotent stem cell derived lung organoids. eLife 4, e05098 (2015)

38. Eiraku, M. et al. Self-organizing optic-cup morphogenesis in three-dimensional culture. Nature 472, 51-56 (2011)

39. Mariani, J. et al. Modeling human cortical development in vitro using induced pluripotent stem cells. Proc. Natl Acad. Sci. USA 109, 12770-12775 (2012).

40. Lancaster, M. A. et al. Cerebral organoids model human brain development and microcephaly. Nature 501, 373-379 (2013).

41. Koehler, K. R., Mikosz, A. M., Molosh, A. I., Patel, D. \& Hashino, E. Generation of inner ear sensory epithelia from pluripotent stem cells in 3D culture. Nature $\mathbf{5 0 0}$ 217-221 (2013)

42. Takasato, M. et al. Kidney organoids from human iPS cells contain multiple lineages and model human nephrogenesis. Nature 526, 564-568 (2015).

43. Valente, M. J. et al. A rapid and simple procedure for the establishment of human normal and cancer renal primary cell cultures from surgical specimens. PLOS ONE 6, e19337 (2011)

44. Xia, Y. et al. Directed differentiation of human pluripotent cells to ureteric bud kidney progenitor-like cells. Nat. Cell Biol. 15, 1507-1515 (2013).

45. Takasato, M. et al. Directing human embryonic stem cell differentiation towards a renal lineage generates a self-organizing kidney. Nat. Cell Biol. 16, 118-126 (2014).

46. Taguchi, A. et al. Redefining the in vivo origin of metanephric nephron progenitors enables generation of complex kidney structures from pluripotent stem cells. Cell Stem Cell 14, 53-67 (2014).

47. Ng, A. \& Barker, N. Ovary and fimbrial stem cells: biology, niche and cancer origins. Nat. Rev. Mol. Cell Biol. 16, 625-638 (2015)

48. Lancaster, M. A. \& Knoblich, J. A. Organogenesis in a dish: modeling development and disease using organoid technologies. Science 345, 1247125-1247129 (2014).

49. Murphy, W. L., McDevitt, T. C. \& Engler, A. J. Materials as stem cell regulators. Nat. Mater. 13, 547-557 (2014).

50. Soen, Y., Mori, A., Palmer, T. D. \& Brown, P. O. Exploring the regulation of human neural precursor cell differentiation using arrays of signaling microenvironments. Mol. Syst. Biol. 2, 37 (2006).
51. LaBarge, M. A et al. Human mammary progenitor cell fate decisions are products of interactions with combinatorial microenvironments. Integr. Biol. 1, 70-79 (2009).

52. Flaim, C. J., Chien, S. \& Bhatia, S. N. An extracellular matrix microarray for probing cellular differentiation. Nat. Methods 2, 119-125 (2005).

53. Fordham, R. P. et al. Transplantation of expanded fetal intestinal progenitors contributes to colon regeneration after injury. Cell Stem Cell 13, 734-744 (2013).

54. Mustata, R. C. et al. Lgr4 is required for Paneth cell differentiation and maintenance of intestinal stem cells ex vivo. EMBO Rep. 12, 558-564 (2011).

55. Mustata, R. C. et al. Identification of Lgr5-independent spheroid-generating progenitors of the mouse fetal intestinal epithelium. Cell Rep. 5, 421-432 (2013).

56. Okamoto, R. \& Watanabe, M. Role of epithelial cells in the pathogenesis and treatment of inflammatory bowel disease. J. Gastroenterol. 51, 1-11 (2015).

57. Sato, T. et al. Paneth cells constitute the niche for Lgr 5 stem cells in intestinal crypts Nature 469, 415-418 (2011).

58. Simmini, S. et al. Transformation of intestinal stem cells into gastric stem cells on loss of transcription factor Cdx2. Nat. Commun. 5, 5728 (2014)

59. Van Es, J. H. et al. DII1+ secretory progenitor cells revert to stem cells upon crypt damage. Nat. Cell Biol. 14, 1099-1104 (2012).

60. Yui, S. et al. Functional engraftment of colon epithelium expanded in vitro from a single adult Lgr5+ stem cell. Nat. Med. 18, 618-623 (2012).

61. Zhang, Y. G., Wu, S., Xia, Y. \& Sun, J. Salmonella-infected crypt-derived intestinal organoid culture system for host-bacterial interactions. Physiol. Rep. 2, e12147 (2014).

62. Horita, N. et al. Fluorescent labelling of intestinal epithelial cells reveals independent long-lived intestinal stem cells in a crypt. Biochem. Biophys. Res. Commun. 454, 493-499 (2014).

63. Wang, X. et al. Cloning and variation of ground state intestinal stem cells. Nature $\mathbf{5 2 2}$ 173-178 (2015).

64. Watson, C. L. et al. An in vivo model of human small intestine using pluripotent stem cells. Nat. Med. 20, 1310-1314 (2014).

65. Van de Wetering, M. et al. Prospective derivation of a living organoid biobank of colorectal cancer patients. Cel/ 161, 933-945 (2015).

66. Greggio, C. et al. Artificial three-dimensional niches deconstruct pancreas development in vitro. Development 140, 4452-4462 (2013).

67. McCracken, K. W. et al. Modelling human development and disease in pluripotent stem-cell-derived gastric organoids. Nature 516, 400-404 (2014).

68. Grun, D. et al. Single-cell messenger RNA sequencing reveals rare intestinal cell types Nature 525, 251-255 (2015).

69. Kusters, J. G., van Vliet, A. H. M. \& Kuipers, E. J. Pathogenesis of Helicobacter pylori infection. Clin. Microbiol. Rev. 19, 449-490 (2006).

70. Wroblewski, L. E. et al. Helicobacter pylori targets cancer-associated apical-junctional constituents in gastroids and gastric epithelial cells. Gut 64, 720-730 (2015).

71. DesRochers, T. M. et al. Effects of Shiga toxin type 2 on a bioengineered threedimensional model of human renal tissue. Infect. Immun. 83, 28-38 (2015).

72. Li, X. et al. Oncogenic transformation of diverse gastrointestinal tissues in primary organoid culture. Nat. Med. 20, 769-777 (2014).

73. Mariani, J. et al. FOXG1-dependent dysregulation of GABA/glutamate neuron differentiation in autism spectrum disorders. Cell 162, 375-390 (2015).

74. Marx, V. Cell-line authentication demystified. Nat. Methods 11, 483-488 (2014).

75. Masters, J. R. \& Stacey, G. N. Changing medium and passaging cell lines. Nat. Protocols 2, 2276-2284 (2007)

76. Gracz, A. D. et al. A high-throughput platform for stem cell niche co-cultures and downstream gene expression analysis. Nat. Cell Biol. 17, 340-349 (2015).

77. Cao, L. et al. Development of intestinal organoids as tissue surrogates: cell composition and the epigenetic control of differentiation. Mol. Carcinogen. 54 189-202 (2015).

78. Meng, Q. Three-dimensional culture of hepatocytes for prediction of drug-induced hepatotoxicity. Expert Opin. Drug Metab. Toxicol. 6, 733-746 (2010).

79. Fukuda, M. et al. Small intestinal stem cell identity is maintained with functional Paneth cells in heterotopically grafted epithelium onto the colon. Genes Dev. 28 1752-1757 (2014)

80. Bertaux-Skeirik, N. et al. CD44 plays a functional role in Helicobacter pylori-induced epithelial cell proliferation. PLoS Pathog. 11, e1004663 (2015).

81. Nadauld, L. D. et al. Metastatic tumor evolution and organoid modeling implicate TGFBR2 as a cancer driver in diffuse gastric cancer. Genome Biol. 15, 428 (2014).

82. Schumacher, M. A. et al. The use of murine-derived fundic organoids in studies of gastric physiology. J. Physiol. 593, 1809-1827 (2015).

83. McCracken, K. W. Howell, J. C. Wells, J. M. \& Spence, J. R. Generating human intestinal tissue from pluripotent stem cells in vitro. Nat. Protoc. 6, 1920-1928 (2011)

84. Ootani, A. et al. Sustained in vitro intestinal epithelial culture within a Wnt-dependent stem cell niche. Nat. Med. 15, 701-706 (2009)

85. Takebe, T. et al. Generation of a vascularized and functional human liver from an iPSCderived organ bud transplant. Nat. Protoc. 9, 396-409 (2014).

86. Chua, C. W. et al. Single luminal epithelial progenitors can generate prostate organoids in culture. Nat. Cell Biol. 16, 951-961 (2014).

87. Koehler, K. R. \& Hashino, E. 3D mouse embryonic stem cell culture for generating inner ear organoids. Nat. Protoc. 9, 1229-1244 (2014).

88. Lancaster, M. A. \& Knoblich, J. A. Generation of cerebral organoids from human pluripotent stem cells. Nat. Protoc. 9, 2329-2340 (2014). 